\title{
Editorial
}

\section{DENTAL AMALGAM PHASE-DOWN}

For more than 150 years, teeth have been restored with dental amalgam. Why this material that has been used for generations have to stop its use? Is it safe for patients and professionals? Is there another alternative material to fulfill patient's needs? Is this material accessible to all the populations? Is it true that amalgam mercury should be dangerous? These are some questions that often appear worldwide.

The Minamata Convention on Mercury is a treaty that opened for signature in October 2013 and provides controls and reductions across a range of products, processes and industries where mercury is used, released or emitted. The FDI World Dental Federation, during the Convention, advocated a reduction (phase-down), instead of a ban of dental amalgam (phase-out) through increased attention to dental prevention and health promotion, increased research and development on alternatives, and best management techniques for amalgam waste.

Besides, efforts of World Health Organization (WHO), the International Association for Dental Research (IADR) and the International Dental Manufacturers (IDM) succeeded in ensuring that dental practitioners would continue to use dental amalgam when needed.

The amount of mercury in amalgam does not represent a health risk to patients. Nevertheless, the exposure to volatile-free mercury in dental office should be controlled to avoid professional contamination. Thus, the use of encapsulated amalgam provides a safe manipulation and correct proportion of the material, as well as decreases the risk of contamination. It has to be mentioned that greater exposure is related to daily mercury intake by food, as consumption of fish with high $\mathrm{Hg}$ levels.

Biomonitoring urinary mercury (U-Hg) helps scientists plan and conduct research on exposure and health effects of mercury contamination. Thereby U-Hg indicates current exposure when mercury reaches the renal steady state and the maximum level allowed for a person is $35 \mathrm{micrograms} / \mathrm{g}$ creatinine.

ADA (2007) developed 'Best management for amalgam waste' a series of practices that should include using chair side traps, installing amalgam separators compliant with ISO 111432, using vacuum collection, inspecting and cleaning traps, collecting and recycling amalgam. It is important that professionals check in their cities whether there is amalgam waste recycling program that complies with ADA-ANSI standard.

Dental amalgam places an important role where there is high caries prevalence, with large cavities associated with poor oral hygiene, remaining the material of choice to re-establish form and function of natural teeth in public healthy care, or low-income communities, because of its longevity, and cost-effectiveness. Moreover, there is enormous scientific evidence proving that dental amalgam is safe and clinically effective, supporting a phase-down rather than a phase-out approach to dental amalgam.

Materials like glass-ionomer cements and resin-based composite materials have benefits such as fluoride release and adhesion, respectively, but are significantly more expensive due to the technical difficulty in fabrication, material costs, high technologies and chairside time required for placement.

FDI supports the WHO recommendation that researchers should continue to investigate even better treatment outcomes for patients; thus, the search for a promising dental restorative care that should substitute dental amalgam continues, in terms of longevity, cost-effectiveness and easy of implementation.

I have found over 20 years of clinical observation (private and public) amalgam restorations in function dating more than 40 years, with corrosion but without any microleakage or secondary caries, that did not needed to be replaced.

It will take some years to improve a direct restorative material that will overcome this advantage of clinical longevity, until that dentists should reduce the frequency of dental amalgam use, restore teeth with amalgam when there is no better possibility, and try to reduce risks, treat and properly dispose amalgam wastes to minimize pollution to environment.

Karen Cristina Kazue Yui

Assistant Professor

São Paulo State University - UNESP

Institute of Science and Technology

Sao Jose dos Campos, Sao Paulo, Brazil 